\title{
Investigation on the Thermal Control Algorithm of the Heat Sink Temperature Regulating System for Thermal Vacuum Tests
}

\author{
Jian Yabin ${ }^{a}$, Zhang Chunyuan, Wang jing, Lin Boyin, Wang Runze and Yin Xiaofang \\ Beijing Institute of Spacecraft Environment Engineering, Beijing 100094, China
}

\begin{abstract}
The heat sink temperature is often set in a semi-empirical way based on steady-state temperatures. As a result, the entrance parameters of the heat sink working fluid are often too conservative, and the rate of temperature rise is difficult to control. In this paper, transient thermal models for each component of the heat sink temperature regulation system are established. Then they are programmed into a dynamic simulation model by using Matlab/Simulink as the thermal control algorithm.It is shown that the model can accurately reflect the dynamic and steady state characteristics of the heat sink temperature regulation system, and can provide guidance for the selection of control strategies and working fluid parameters in the thermal vacuum test.
\end{abstract}

\section{Introduction}

The thermal vacuum test is to assess the performances and to expose the design and process problems of spacecraft components, subsystems or the whole spacecraft under extreme hot and cold vacuum conditions. In thermal vacuum tests, the use of heat sink temperature regulation has the advantages of economy, convenient operation, fast cooling, and strong adaptability to complex shape and moving parts. It is an important development direction of spacecraft component level thermal vacuum test[1-3]. At present, in the process of heat sink temperature regulation, the initial temperature, the final temperature and the cooling time usually are taken to be the known conditions. The temperature of the heat sink is set by a semi-empirical method based on steady-state model. The parameters of the inlet working fluid are regulated automatically by PID. Due to the large lag caused by the heat capacity of the heat sink, the inlet parameters obtained by steady state calculation are usually much more conservative than the actual conditions during the test. The heat sink temperature regulation time is too long, and the rate of temperature rise and fall of the test article is difficult to control [4].

In the past, the research of heat sink temperature control system mainly focused on heat sink structure and external cryogenic system. There was less research on the dynamic temperature characteristics of the whole system, and the thermal model of the test article was relatively simple [5-6]. Here, based on transient thermal model, the heat transfer relationship of the whole heat sink temperature regulating system is analyzed, and a dynamic simulation model is established by Matlab/Simulink. The dynamic response relationship among the entrance working fluid parameters, the heat sink and the test article is obtained. The model is programmed into the

a Corresponding author : cast5118@163.com 
temperature control algorithm for the test article, and applied in the thermal vacuum tests. The results show that the established dynamic model and simulation program can reflect the steady and dynamic characteristics of the test article accurately, which is of great significance for the formulation of the test strategy, the selection of test parameters, and the prediction of the test process.

\section{Model of heat sink temperature regulating system}

The heat transfer of heat sink can be divided into the inner part and the outer part. There are two paths for the outer heat transfer, one is to the test article, the other is to the wall of the chamber (including radiation shield). In these two paths heat is exchanged by thermal radiation. For the inner part, heat is exchanged with the working material by convection heat transfer. Based on the idea of object-oriented programming and parametric modeling, the simulation system is composed of 3 separate modules: heat sink heat exchanger module, test article module and radiation heat transfer module [5].

\subsection{Heat sink heat exchanger module}

The heat sink is a pipe network system composed of finned tubes. For the convenience of modeling, one of the single branch pipes was analyzed. The branch was divided into $n$ nodes, and the length of the microelement $d l$ was taken as the control body. Based on energy conservation, the change of energy in the control body is equal to the algebraic sum of heat flux in and out. The temperature of each control body in the tube wall at the $\tau$ moment can be expressed as follow:

$$
\begin{gathered}
\Delta m \cdot c \cdot \frac{d T_{i}}{d \tau}=\delta q+h_{i} S_{1}\left(T_{i}^{\prime}-T_{i}\right) \\
\delta q=q / k / n \\
q=q_{1}+q_{2}
\end{gathered}
$$

Where $q_{1}$ is radiation heat transfer between heat sink and test article; $q_{2}$ is radiation heat transfer between heat sink and chamber; $k$ is the number of branch pipes; $n$ is the number of nodes of one branch pipe; $\Delta m=M / k / n$ is mass of the control body; $M$ is mass of heat sink; $T_{i}^{\prime}$ is temperature of the fluid at the node $i ; T_{i}$ is temperature of the heat sink at the node $i ; c$ is the average heat capacity of heat sink; $S_{1}$ is convective heat transfer area between heat sink and working fluid.

Using the fitted equations of refrigerant properties[6] and the heat transfer correlations[7-9], The convective heat transfer program can be written, and then it can be executed to dll files using the mex command. Through the S-function, the convective heat transfer calculation module can be given as shown in figure 1. The input is external heat flux, and the output is the average temperature of the heat sink .

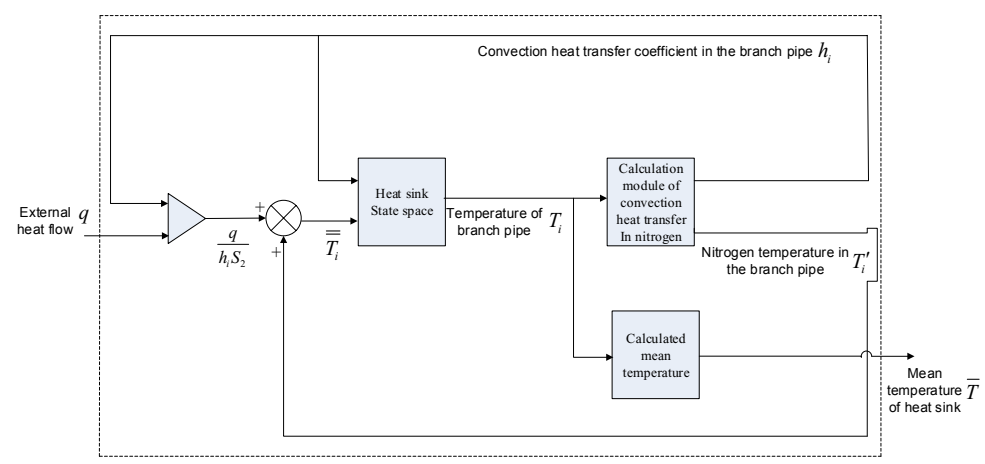


Figure 1. Heat exchange module of heat sink-working fluid

The parameter needed by the simulation can be a single variable or variables in a form of a matrix.

\section{2 test article module}

The test article is simplified as a solid cylinder with the same material, mass and surface area as the actual test article. It is treated as one-dimensional heat conduction. This simplification is feasible for most engineering applications .

The structure of the test article is divided into $m$ layers, as shown in Figure 2.

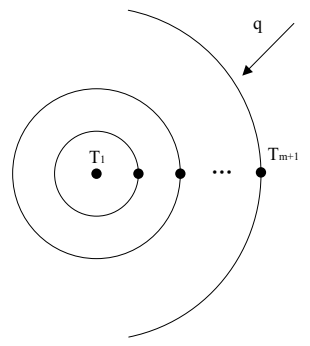

Figure2. Simplified heat transfer model of test article

The one-dimensional heat conduction model of the cylindrical object is discretized by the two order formulation [8].

$$
C \frac{d T_{i}}{d \tau}=\frac{T_{i-1}-2 T_{i}+T_{i+1}}{R}-\frac{T_{i-1}-T_{i+1}}{2(i-1) R}
$$

where $C$ is specific heat capacity of test article; $R=\Delta r / \lambda$ is the thermal resistance.

For the $m+1$ point, which is on the outer boundary, the energy change in the control body is equal to the heat transferred from the $m$ point added the heat transferred from the outside, which can be simplified to:

$$
\frac{d T_{m+1}}{d \tau}=\frac{2(m-1 / 2)}{R C(m-1 / 4)}\left(T_{m}-T_{m+1}\right)+\frac{\rho c_{p}}{C^{2} L \pi(m-1 / 4)} q
$$

When the difference between the average temperature of the test article and the outside surface is small, it is equivalent to the lumped parameter method ignoring the internal thermal resistance of the test article..

The module take the radiative heat flux as the input, and output the temperature of the test article.

It can be selected whether or not to treat the test article by lumped parameter method. When the lumped parameter method is selected, only the correlation parameters and initial temperature of the test article are required for input. When the lumped parameter method is not selected, the parameters such as the test article 's density and thermal conductivity coefficient should also be input.

\subsection{Calculation module of radiation heat transfer}

The heat transfer between the heat sink and other surfaces (the chamber and the test article) can be regarded as the typical radiation heat transfer: thermal radiation among isothermal grey surfaces.

The mathematical model of radiation heat transfer is provided by Simulink, it is built and encapsulated into a radiation heat transfer subsystem to form a radiation heat exchange module. 


\subsection{Simulation model of heat sink and temperature regulating system}

According to the modules established above, the schematic diagram of the system simulation model can be built,as shown in Figure3.

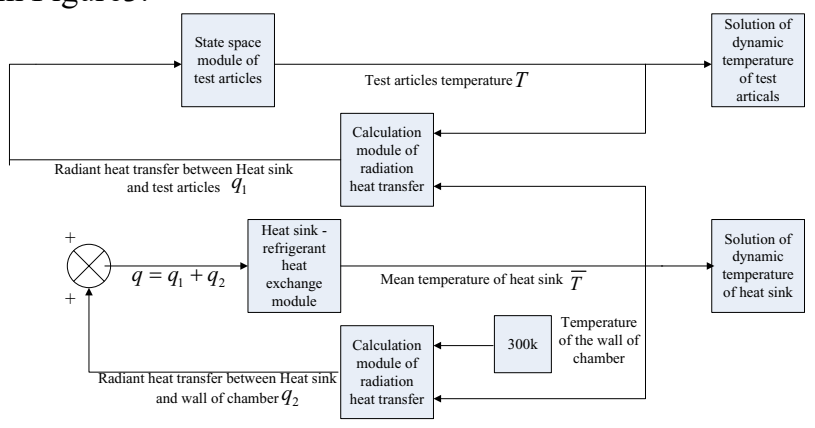

Figure3. Schematic diagram of heat sink temperature regulating system simulation model

For a small vacuum chamber, the entrance pressure of the working fluid (nitrogen pressure) is $0.4 \mathrm{Mpa}$, the entrance temperature is $150 \mathrm{~K}$, the total flow rate is $1.5 \mathrm{~m}^{3} / \mathrm{min}$. The temperature variation of the heat sink branch pipe nodes $(\mathrm{k}=50)$ in 3600 seconds are shown in Figure 4.

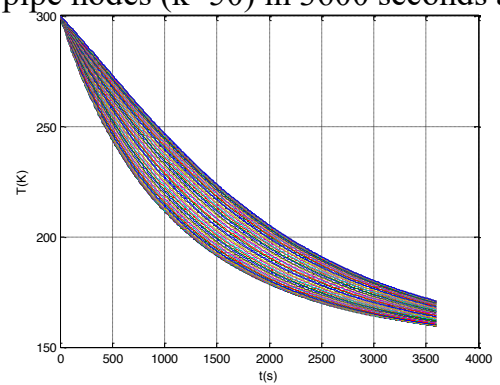

Figure4. Response curve of heat sink temperature

The test article is a electro-thermal component with mass $10 \mathrm{~kg}$, surface area $5 \times 10^{-3} \mathrm{~m}^{2}$, internal heat source $20 \mathrm{~W}$, and surface emissivity 0.9 . The temperature variation during heat sink cooling is shown in Figure 5.

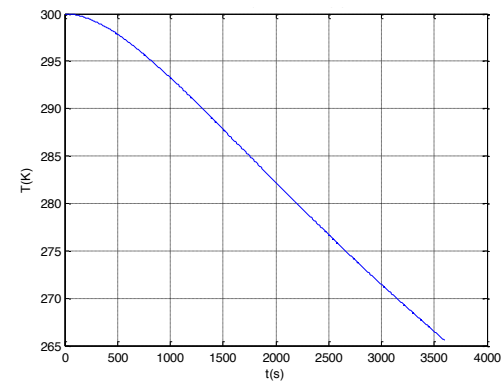

Figure5. Response curve of test article temperature

This dynamic simulation model is superior to the traditional model in that it predicts a temperature variation agreed well with the actual cooling process.

\section{Temperature control algorithm based on transient thermal model}


Using the Matlab external commands, the simulation model can calculate the steady and dynamic parameters of the heat sink, the test article and the entrance working fluid parameters. It can also do the reverse operation to calculate the entrance working fluid parameters which satisfy the requirement of the target temperature and the temperature change rate of heat sink or the test article. In the following, the dynamic calculation is performed, and it is illustrated how to guide the setting of the entrance working fluid parameters of the thermal vacuum test.

During the thermal vacuum test, the temperature change rate of the test article is often specified. For the simulation model established, the temperature change rate is solved in two ways: "Positive calculation" and "Inverse calculation". "Positive calculation" is to calculate the temperature change rate of the heat sink or the test article given the parameters of the heat sink entry. "Inverse calculation" is to calculate the inlet variables of the heat sink to satisfy the temperature rate. In practice, the "Inverse calculation" is of more practical value.

Using SIM command, provided by Matlab, we can get the output of the system at any time under various input conditions. In the thermal vacuum test, temperature variation of the heat sink and test article can be obtained through the SIM command effectively.

The key to calculating the temperature change rate("Positive calculation") is to get the time duration required for the system to reach the target temperature. The result of the SIM command can be seen as a "function" of the input time variable. The time required is regarded as the solution equation of the function. A secant method is used to calculate the time required to reach a certain temperature. The program is saved as searcht.m to be called during the "Inverse calculation" process of the temperature change rate.

For a single input system, there is only one demarcation point that meets the requirements of the temperature change rate. The solution of the point $T_{0}$ can be carried out according to the following algorithm: First, the trim command provided by Matlab is used to calculate the heat sink inlet temperature $T_{s}$, which can make the heat sink stable at the target temperature $T_{2}$. Then the average temperature change rate $\kappa^{\prime}$ of the heat sink from the initial temperature $T_{1}$ to the target temperature $T_{2}$ is calculated at the inlet working fluid temperature $T_{0}=T_{s}$. If $\kappa^{\prime}<\kappa$, then $T_{0}=T_{0}-1 K$, if $\kappa^{\prime}>\kappa$, then $T_{0}=T_{0}+1 K$, until the boundary point of the condition is satisfied.

Generally, The inlet parameters calculated by the target stable temperature are difficult to meet the requirement of temperature change rate. The program diagram for solving the parameters of the heat sink entrance to meet the temperature change rate of the test article is shown in Figure 6. 


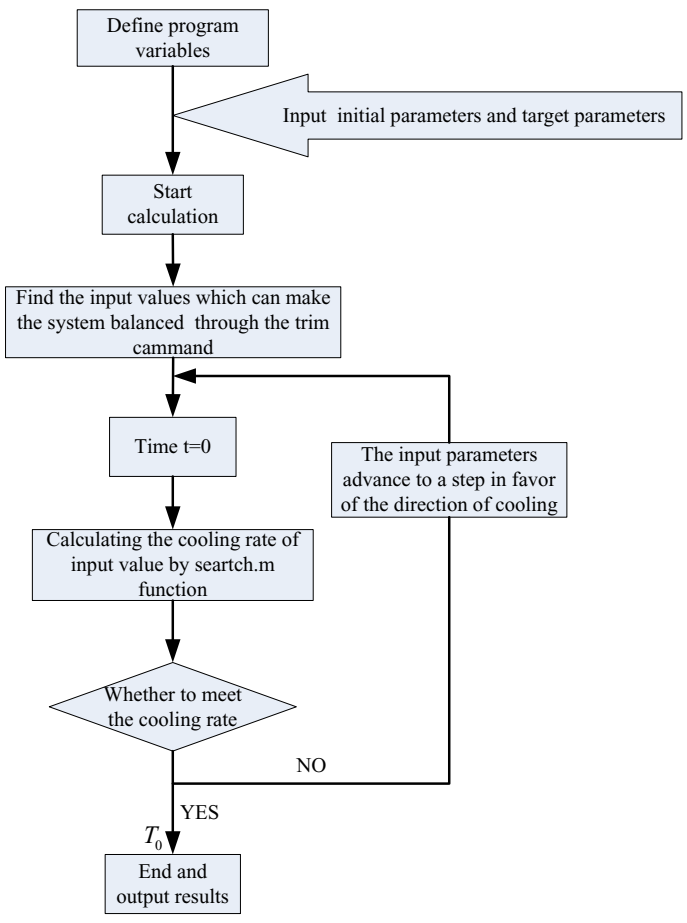

Figure6. Block diagram of cooling rate

The above entry parameters can be the flow or temperature of the heat sink inlet working fluid.

Combined with a test equipment, the average cooling rate of the test article from $30^{\circ} \mathrm{C}$ to $-60{ }^{\circ} \mathrm{C}$ is not less than $0.5^{\circ} \mathrm{C} / \mathrm{min}$, and the temperature is eventually stabilized at $-60^{\circ} \mathrm{C}$. The compressor outlet pressure is $0.4 \mathrm{Mpa}$, the heat sink inlet flow rate is $5 \mathrm{~m} 3 / \mathrm{min}$ (standard condition), In this case, the heat sink inlet temperature can be calculated to meet the cooling rate and the target temperature of the test article.

Input the known parameters and initial parameters in the interface, select "control test article" in the drop-down menu of "select control target" ("control test article" or "control heat sink"). "-0.5" stands for the cooling rate is $0.5^{\circ} \mathrm{C} / \mathrm{min}$. In the choice of " heat sink entrance conditions "(" flow "or" temperature ") pull-down menu ,select" flow ", standing for the flow of the inlet working fluid is known. The simulation curve calculated by the program is shown in Figure7.

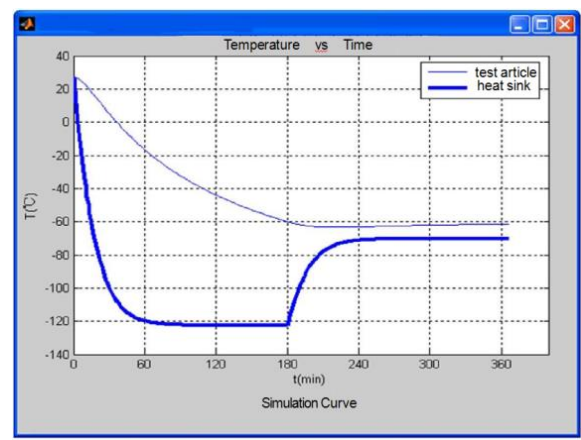

Figure7. The simulation curve interface of model

The inlet flow rate of the heat sink $\mathrm{Qv}$ is $5 \mathrm{~m}^{3} / \mathrm{min}$, the inlet temperature $T_{0}$ is $-124.9^{\circ} \mathrm{C}$. Then the temperature of the test article is reduced from the initial temperature to $-60^{\circ} \mathrm{Cat} \mathrm{t}=180 \mathrm{~min}$, Meeting 
the requirement of cooling rate. At the end of the cooling stage, the inlet flow rate is constant, the temperature is changed to $=-71.8{ }^{\circ} \mathrm{C}$, The final temperature of the test article is finally stabilized at $-60{ }^{\circ} \mathrm{C}$. the final temperature of the heat sink is $-70.3{ }^{\circ} \mathrm{C}$.

\section{Conclusion}

In this paper, mathematical models for the heat transfer of the working fluid, heat sink and test article are established. They are integrated into the heat sink temperature regulating system. Then the Simulink model of the overall heat sink temperature control simulation system are built, and the temperature control algorithm based on the transient thermal model is realized. It can not only simulate the steady and dynamic simulation with the various input parameters, but also can carry out the "Inverse calculation" under certain test conditions to provide guidance for the parameter setting of the heat sink temperature regulating system in the thermal vacuum test.

\section{References}

1. HUANG Bencheng, Environment test technology of Spacecraft [M].,2002:60-98

2. SHAO Xingguo, LIU Zijun, TAN Changhai,et a1. Research on heat flux measurement and simulation in thermal balance test of spacecraft[J]. Spacecraft Engineering,2007,16(4): 94-98

3. Andrina G, Cane L, Panelh B. Solar Simulation with Infrared Techniques Applicability to Mercury/ Bepicolombo Probe System Thermal Test. Proceedings of 4th International Symposium on Environmental Testing for Space Program, ESTEC, Noordwijk Netherlands. 2001:327-332

4. Michael T. Fournier. Controller for an environment test chamber. SCI, United States Patent NO. US6,023,985, 2000,1

5. JIAN Yabin, ZHANG Chunyuan, DING Wenjing, et al. Simulation design based on state-pace for regulating heat sink[J] Spacecraft Environment Engineering,2008,25(4):373-376

6. JIANG Chansheng. Dynamic Simulation Technology for Cryogenic Shrouds in Large Space Simulators [D].Beihang University,1999

7. Yakhot V, Orzag S A. Renormalization group analysis of turbulence:basic theory[J].Journal of Scientific Computing, 1996(1):3-11

8. LI Qingfang, ZHANG Guozhong, LIN Yuanyuan,et al.Study of heattransfer and pressure drop in tubes with rectangle-like fins[J].Journal of Engineering Thermophysics, 2009,30(12):2122-2124

9. LUO Baojun, WANG Zhaoli, YAN Tao,et al. Design and Implementation of a Space Sub-KelVin Sorption Cooler[J]. Journal of Astronautics,2015,36(7):855-859 